\title{
Moving a rubber hand that feels like your own: a dissociation of ownership and agency
}

\section{Andreas Kalckert* and H. Henrik Ehrsson}

Brain, Body and Self Laboratory, Department of Neuroscience, Karolinska Institutet, Stockholm, Sweden

Edited by:

Burkhard Pleger, Max Planck Institute

for Human Cognitive and Brain

Sciences, Germany

\section{Reviewed by:}

Marco Taubert, Max Planck Institute for Human Cognitive and Brain

Sciences, Germany

Mel Slater, University of Barcelona,

Spain

Peter Schwenkreis,

Universitaetsklinikum Bergmannsheil, Germany

Roger Newport, University of

Nottingham, UK

${ }^{*}$ Correspondence:

Andreas Kalckert, Department of

Neuroscience, Karolinska Institutet,

Retzius väg 8, 17177 Stockholm,

Sweden.

e-mail: andreas.kalckert@ki.se
During voluntary hand movement, we sense that we generate the movement and that the hand is a part of our body. These feelings of control over bodily actions, or the sense of agency, and the ownership of body parts are two fundamental aspects of the way we consciously experience our bodies. However, little is known about how these processes are functionally linked. Here, we introduce a version of the rubber hand illusion in which participants control the movements of the index finger of a model hand, which is in full view, by moving their own right index finger. We demonstrated that voluntary finger movements elicit a robust illusion of owning the rubber hand and that the senses of ownership and agency over the model hand can be dissociated. We systematically varied the relative timing of the finger movements (synchronous versus asynchronous), the mode of movement (active versus passive), and the position of the model hand (anatomically congruent versus incongruent positions). Importantly, asynchrony eliminated both ownership and agency, passive movements abolished the sense of agency but left ownership intact, and incongruent positioning of the model hand diminished ownership but did not eliminate agency. These findings provide evidence for a double dissociation of ownership and agency, suggesting that they represent distinct cognitive processes. Interestingly, we also noted that the sense of agency was stronger when the hand was perceived to be a part of the body, and only in this condition did we observe a significant correlation between the subjects' ratings of agency and ownership. We discuss this in the context of possible differences between agency over owned body parts and agency over actions that involve interactions with external objects. In summary, the results obtained in this study using a simple moving rubber hand illusion paradigm extend previous findings on the experience of ownership and agency and shed new light on their relationship.

Keywords: sense of ownership, sense of agency, rubber hand illusion, self-recognition, body perception, multisensory integration, volition, voluntary movement

\section{INTRODUCTION}

In normal situations, we all perceive that our body is our own and that we can control its movements voluntarily. If you ask a child "can you move your hand?," the child will likely reply "of course I can move my hand." We do not usually question the experience of the body as a part of the self or the fact that we control the actions of our bodies, even as young children. It has been argued in various fields, such as psychology, philosophy and cognitive science, that these particular experiences of one's own body are of critical importance to the conscious experience of the self (Merlau-Ponty, 1945; Chiel and Beer, 1997; Gallagher, 2000, 2005; Churchland, 2002; Graziano and Botvinick, 2002; Jeannerod, 2003; Haggard, 2005). Current cognitive neuroscience research indicates that these two basic aspects of an individual's self-perception of the body are critically important for self-consciousness: the experience of the body as one's own, which is referred to as the sense of "body ownership" (Gallagher, 2005; Makin et al., 2008; Ehrsson, 2012), and the sense of authorship over bodily movements, termed the sense of "agency" (Blakemore and Frith, 2003; Haggard, 2005; Gallagher, 2007; David et al., 2008; Synofzik et al., 2008a). The experience of the movement of a body part that is not self-generated indicates the presence of an external force, which in turn may imply a potential threat, such as being grabbed by an enemy. Similarly, manual interactions with environmental objects and the generation of defensive actions to avoid potential threats require the ability to identify and localize the limbs in space (Graziano and Cooke, 2006). Thus, from an evolutionary perspective, the abilities that allow us to experience the causal origin of actions and sense the boundary between one's body and the external world are crucial. However, in the majority of everyday situations, we experience the senses of ownership and agency as intimately coupled. How are these processes structured and how do they interact? Are the sensations of ownership and agency two fundamentally different processes, or might a single process explain both of these bodily experiences?

The sense of ownership is assumed to rely on the integration of sensory signals from different modalities (Botvinick, 2004; Makin et al., 2008; Ehrsson, 2012) or so-called "multisensory integration" (Holmes and Spence, 2005; Stein and Stanford, 2008). When the spatial, temporal, visual and somatic signals received from a limb all match, a feeling of ownership then arises for this limb. 
This phenomenon has been demonstrated convincingly in studies investigating the rubber hand illusion (Botvinick and Cohen, 1998). In this illusion, a rubber hand is placed in full view on a table in front of the participant and touched in synchrony with touches applied to the participant's hand, which is hidden behind a screen. After a short period of such repetitive visuo-tactile stimulation, the majority of participants begin to feel the touch where they see it on the rubber hand rather than where it is applied to their real hand and develop a feeling that the rubber hand is their own hand. Asynchronous stimulation of the two hands abolishes the illusion (Shimada et al., 2009b), as does placing the rubber hand in an anatomically implausible position (Ehrsson et al., 2004; Tsakiris and Haggard, 2005). Hence, both a temporal correlation between visual and tactile signals and a match between the visual and perceived static positions of the hands are necessary to elicit the illusion of ownership (Tsakiris and Haggard, 2005; Makin et al., 2008; Ehrsson, 2012).

How is the sense of agency over our movements produced? Frith et al. (2000) suggested that the comparator/central monitoring process used for the optimal sensorimotor control of action (Wolpert and Miall, 1996; Wolpert and Ghahramani, 2000) may also be responsible for generating the sense of agency. This model is based on the premise that every voluntary movement is accompanied by an efferent copy, which is used to compute the expected sensory consequences. The comparison of the efferent copy with the actual feedback thus enables the agent to distinguish between internally and externally generated sensations (von Holst and Mittelstaedt, 1950; Bays et al., 2005; Crapse and Sommer, 2008). If the efferent copy and feedback match, then the movement has been performed as intended. If the perceived feedback clearly violates the expected outcome, then the participant becomes aware of this discrepancy and does not experience authorship of the action (van den Bos and Jeannerod, 2002; Farrer et al., 2008b). Disturbances of this comparator process have been suggested to underlie the abnormal experience of movements in pathological conditions (Frith et al., 2000; Franck et al., 2001; Blakemore and Frith, 2003; Shergill et al., 2005; Voss et al., 2010).

However, it has also been argued that the sense of agency has a more complex structure, with multiple levels, presumably involving different processes (for further discussion, see Haggard et al., 2002; Jeannerod, 2003; David et al., 2008; Synofzik et al., 2008a,b). In particular, motor intentions, which are potentially related to the direct, conscious experience of preparatory motor commands in premotor areas (Lau et al., 2004, 2007), may also be crucial to the sense of agency (e.g., Haggard et al., 2002; Haggard, 2005). Moreover, matching between the intention to act and the perception of action goals has also been proposed to be a general mechanism supporting the sense of agency (Wegner et al., 2004; Sato, 2009).

Most cognitive neuroscience studies of agency make no fundamental distinction between the simple movements of limbs and goal-directed actions that involve tool use or manual interactions with objects in the environment (such as lifting a cup of tea). Indeed, the arbitrary mapping of motor intentions and sensory events can be learned (Sato and Yasuda, 2005; Sato, 2009; Spengler et al., 2009) and thereafter predicted, and novel internal models can be acquired to facilitate tool use (Imamizu et al., 2000; Wolpert et al., 2011), extending the principles of the comparator model to object-directed actions. Nevertheless, one might ask whether the agency of simple movements of one's body and the agency of changes in the sensory environment resulting from bodily actions actually correspond to the same process. When you raise your arm, you experience your will as being the causative agent of the arm's motion. This type of agency over one's body (hereinafter referred to as "body agency") was described by Wittgenstein in his famous analysis of the phenomenology of willed acts (Wittgenstein, 1958). However, unlike "body agency," agency during interactions with environmental objects and during tool use is not necessarily confined to parts of one's body, and an initial learning period is required for the arbitrary mapping of action and external effect (hereinafter referred to as "external agency"). Many contemporary studies, however, do not address the potential differences between body agency and external agency, and unsurprisingly, the various approaches in the study of agency differ in this particular aspect (Gallagher, 2007; David et al., 2008).

Previous studies aiming to investigate the ownership of moving hands have used video recording technology, in which the motion of the participant's hand is recorded and images of this moving hand are displayed on a screen in front of the participant (Tsakiris et al., 2006). However, Ijsselsteijn et al. (2005) reported significantly weaker ratings of ownership with 2D images of hands projected onto a screen than with the classic illusion involving a physical model of the hand. More recent studies employing sophisticated virtual reality technology(Slater et al., 2008, 2009; Sanchez-Vives et al., 2010) or advanced on-line image manipulation software, such as the Mirage system (Newport and Preston, 2010; Newport et al., 2009), suggest that it is possible to elicit feelings of ownership with moving virtual hands. However, the extent to which the effects of the classic rubber hand illusion can be fully reproduced with areal moving model hand without the use of advanced technology remains somewhat unclear. It is thus important to examine if this is the case as a central prediction of multisensory models of body ownership (Ehrsson, 2012) is that correlations between somatic and visual feedback during natural finger movements should also produce ownership sensations, and we cannot assume that the results obtained from virtual reality simulations will always extend to physical reality because the technology used may influence the reported percepts. One important exception is a study by Dummer et al. (2009; see also the recent "rubber finger" experiment by Walsh et al., 2011), who described experiments in which participants gave high ratings of ownership when a physical model of the moving hand was used. Unfortunately, in this study, no objective evidence for the illusion was reported.

A more serious limitation of the previous studies is that they were not designed to directly dissociate ownership and agency in a single rubber hand illusion paradigm (Tsakiris and Haggard, 2005; Dummer et al., 2009; Kammers et al., 2009; Longo and Haggard, 2009). Tsakiris et al. (2006) compared the strength of ownership, as measured by proprioceptive drift alone, in videobased conditions with active movements, passive movements, or a version of the classic rubber hand illusion in which relaxed hands were brushed. The rationale behind this approach was to examine whether the sense of agency, which is present during active (ownership and agency) but not passive movements (ownership 
only), promoted ownership. The authors reported similar levels of proprioceptive drift in the three conditions when synchronous stimulation was compared with the asynchronous control conditions. In this design, agency was always present in the context of ownership; in other words, no condition with agency without ownership was included. Therefore, it was not possible to test for double dissociation between ownership and agency or to examine possible differences between agency over one's limbs and external objects.

In the present study, we introduce a version of the rubber hand illusion in which participants "move" the index finger of a physical (wooden) model hand by moving their own index finger and describe how this paradigm results in a strong illusion of ownership, quantified by both subjective and objective measures. In the first series of experiments (Experiments 1 and 2), we manipulated the relative timing of visual and somatic feedback. As synchrony is known to play crucial roles in both the sense of ownership and the sense of agency, we hypothesized that asynchronous sensory feedback would effectively eliminate both ownership and agency and thereby serve as a control condition.

In a second series of experiments (Experiments 3 and 4), we tested the hypothesis that ownership and agency represent different processes and can therefore be dissociated. To this end, the mode of movement generation (active versus passive) and the position of the model hand with respect to the participant's body (anatomically congruent versus incongruent positions) were manipulated in a $2 \times 2$ factorial design. Our prediction was that passive movements would eliminate agency but have no influence on ownership, whereas an anatomically incongruent hand posture would eliminate ownership but leave agency intact. In the context of the last prediction, we reasoned that because agency can be experienced during tool use and object-directed actions, it should also be maintained when moving a finger that does not feel like a part of one's body. Our results support our hypotheses and provide evidence of a (double) dissociation between ownership and agency. Furthermore, our factorial design allowed us to directly compare the agency sensed over an owned limb (body agency) with the agency sensed over an external object (external agency). Our results suggest that agency over a part of one's body is more vivid and more tightly linked with ownership than agency over an external object. This finding provides preliminary support that body agency and external agency may involve different processes.

\section{MATERIALS AND METHODS SUBJECTS}

A total of 104 healthy, experimentally naïve participants were tested in four separate experiments. Experiment 1 included 20 participants (nine males, mean age 24.5 years, $S D \pm 4.4$ ). In Experiment 2, another 20 participants were tested (eight males, mean age 30.5 years, $S D \pm 12.6$ ). The third and fourth experiments each involved testing 32 participants (Experiment 3: 15 males, mean age 24.8 years, $\mathrm{SD} \pm 5.8$; Experiment 4:14 males, mean age 27.2 years, $\mathrm{SD} \pm 8.1)$. No volunteers participated in more than one experiment. All participants provided written informed consent prior to participation. The study methodology was approved by the Regional Ethical Review Board of Stockholm (www.epn.se).

\section{APPARATUS AND PROCEDURES}

Participants sat at a table and put their right hand into a wooden box (see Figure 1; dimensions $35 \mathrm{~cm} \times 25 \mathrm{~cm} \times 12 \mathrm{~cm}$ ) placed $30 \mathrm{~cm}$ in front of them. The participant sat comfortably in this position, with the right hand and arm outstretched so that the tip of the index finger was approximately $50 \mathrm{~cm}$ from the shoulder. A life-sized wooden model of a human hand was placed above the box. The model hand measured $20 \mathrm{~cm}$ in length (from the end of the wrist to the tip of the middle finger) and was covered with a latex glove. Subjects wore identical latex gloves on their right hands. A plastic finger cap was placed on the tip of the index finger, which was mechanically connected to the index finger of the model hand above the box by a thin wooden rod installed through a small hole in the box. A blanket was placed over the participant's right shoulder to cover the space between the model hand and the participant and to create a visual scenery for the participant that the model hand was the participant's own outstretched hand (see Figure 1).

Prior to the experiments, the participants were asked to read written instructions that introduced them to the procedure. The participants' task was to tap their right index finger in a semiregular rhythm at approximately $1 \mathrm{~Hz}$. In the passive condition, however, participants were instructed only to relax their fingers (see Experiments 3 and 4 below for further details). A strictly regular rhythm was avoided because such perfectly regular visuosomatic correlations were considered to produce weaker illusions than more irregular patterns (based on anecdotal observations). Thus, in the present experiment, participants were instructed at random intervals to occasionally execute a quick "double tap" instead of a single tap. Participants were briefly trained before the experiment to perform the tapping in the requested manner, using a metronome for pacing. The metronome was not present during the experimental trials. During the experiments, the participants were instructed to look at the model hand and focus on the moving model finger.

The experimenter sat opposite the participant and moved the model hand in the asynchronous and passive conditions (see below). The experimenter's arm was hidden under a cover so

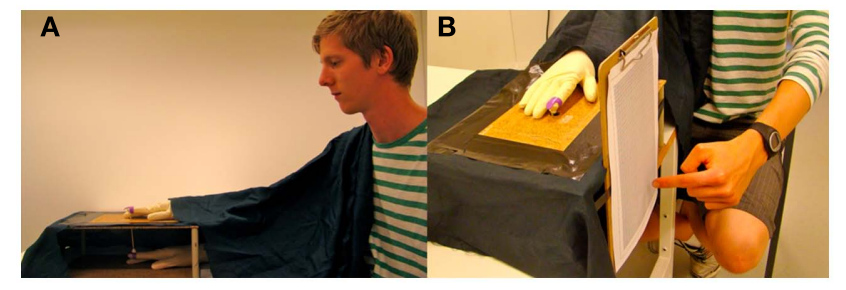

FIGURE 1 | (A) The setup used to induce the moving rubber hand illusion. The participant placed his right hand wearing a latex glove into the box. A wooden model hand wearing an identical latex glove was placed on top of the box. A blanket covered the space from the participant's right shoulder to the right wrist of the model hand. The index fingers of the participant's hand and the model hand were mechanically connected by a rod attached to two small plastic rings on the index fingers. (B) The proprioceptive drift measure. With eyes closed, the participants indicated where they felt their right index finger was located by moving their left index finger to the corresponding location on the board. 
that the participant could not see when the experimenter moved the model index finger. Indeed, post-experimental debriefings revealed that the participants did not develop explicit knowledge of the mechanism responsible for the movements of the model hand. After each trial, the experimenter removed the finger cap and instructed the participant to withdraw the hand from the box, move his right hand and arm to completely break the illusion and then relax for approximately 30-45 s.

\section{CONDITIONS}

In the first two experiments ( 1 and 2 ), the temporal congruence between the real movements of the index finger of the participant and the model hand was manipulated (synchronous versus asynchronous conditions). In the asynchronous condition, the connecting rod was detached from the participant's finger cap. The experimenter now moved the index finger of the model hand by moving the rod manually and introduced an approximately $500 \mathrm{~ms}$ delay with respect to the participant's movement so that the real and artificial fingers appeared to be moving in opposite phases.

In Experiments 3 and 4, a $2 \times 2$ design was applied using the factors movement mode (active versus passive) and model hand position (congruent versus incongruent). Thus, the participants experienced four different experimental conditions: active congruent, active incongruent, passive congruent, and passive incongruent. In these four conditions, the observed movements of the model hand and the movements of the real finger were always synchronous. During the passive movements, the mechanical connection between the model finger and participant's finger was maintained, and the experimenter moved this connection out of the view of the participant. Thus, the index finger of the rubber hand and the participant's passive index finger moved up and down simultaneously in response to an external force. The participants were instructed to relax their fingers during this procedure and not actively participate in the generation of the movements. The experimenter could verify that the participant was complexly relaxed by a monitoring the lack of active or reactive forces in the mechanical device when pulling it up and down. In the incongruent trials, the model hand was rotated $180^{\circ}$ (as in Ehrsson et al., 2004) while maintaining the mechanical connection between the fingers. Importantly, when the model hand was rotated, the position of the wooden index finger was kept constant to ensure a constant viewing angle and distance from the participant.

In the passive and asynchronous movement conditions, the experimenter copied the participant's movements in terms of amplitude, frequency, and number of double taps to mimic the characteristics of the participant's movements from previous trials or the practice session before the experiment.

\section{MEASURES OF THE ILLUSION Questionnaires}

In Experiments 1 and 3, we used a 16-statement questionnaire (Table 1). Four statements referred to the feeling of ownership (e.g., "I felt as if I was looking at my own hand"), and four statements described sensations related to agency (e.g., "I felt as if was causing the movement I saw"). These statements were adopted from existing questionnaires used in traditional rubber hand illusion experiments (e.g., Botvinick and Cohen, 1998; Longo et al.,
Table 1 | The questionnaire, consisting of 16 statements divided into four different categories.

\section{Category}

Ownership

1. I felt as if I was looking at my own hand*

2. I felt as if the rubber hand was part of my body

3. It seemed as if I were sensing the movement of my finger in the location where the rubber finger moved

4. I felt as if the rubber hand was my hand*

Ownership control

5. I felt as if my real hand were turning rubbery

6. It seems as if I had more than one right hand*

7. It appeared as if the rubber hand were drifting

towards my real hand

8. It felt as if I had no longer a right hand, as if my right hand had disappeared*

Agency 9. The rubber hand moved just like I wanted it to, as if it was obeying my will*

10. I felt as if I was controlling the movements of the rubber hand

11. I felt as if I was causing the movement I saw 12. Whenever I moved my finger I expected the rubber finger to move in the same way*

Agency control 13. I felt as if the rubber hand was controlling my will 14. I felt as if the rubber hand was controlling my movements*

15. I could sense the movement from somewhere between my real hand and the rubber hand*

16. It seemed as if the rubber hand had a will of

its own

Asterisks indicate questions used in the shorter version of the questionnaire that was used in Experiments 2 and 4.

2008). The remaining eight statements were control statements, with four for ownership and four for agency (e.g., "I felt as if I had more than one right hand" and "It seemed as if the rubber hand had a will of its own"). These served as controls for task compliancy, suggestibility, and expectancy effects. The control statements were created based on similar control statements used in earlier studies of the rubber hand illusion (e.g., Botvinick and Cohen, 1998; Petkova and Ehrsson, 2008) in that they include statements that bear several similarities to the illusion-specific statements (e.g., includes the word "will" or "hand") but do not capture the phenomenological experiences of ownership or agency. Participants were exposed to $3 \mathrm{~min}$ of stimulation for each individual condition, after which they reported their subjective experience on a 7 -point Likert scale ranging from " -3 " (totally disagree) to " +3 " (totally agree), with " 0 " indicating neither agreement nor disagreement ("uncertain"). In Experiments 2 and 4, we applied a shorter version of the questionnaire to confirm the subjective experience of ownership and agency in these groups of participants. Here, we only included the most important statements related to the perceptions of ownership and agency, i.e., those that had displayed clear and reliable differences in the previous experiments, with the aim of examining possible correlations between the illusion categories and proprioceptive drift (see Table 1). 
To analyze the questionnaire data, the average of the scores for the four statements related to ownership was computed to obtain a single "ownership statement" score. Similarly, the "agency statement" score was defined as the mean score of the four statements related to the sense of agency. The four control statements for ownership and agency were also averaged to obtain "ownership control statement" and "agency control statement" scores, respectively. Thus, references in the text to "ownership statements" or "agency statements" always refer to the average scores of the four individual statements in the original questionnaires unless explicitly stated otherwise. The ownership and agency statement scores were compared with their corresponding control statements. An average rating $\geq+1$ indicates that on the group level, the participants affirmed the statement, i.e., they had experienced ownership or agency (this criterion has been used before; see Ehrsson et al., 2004; Petkova and Ehrsson, 2009).

\section{Proprioceptive drift measure in the pointing task}

Experiments 2 and 4 measured the degree of erroneous pointing toward the model hand when the participants were asked to indicate the perceived location of their right index finger. This indication served as an objective behavioral measure of the illusory feeling of ownership over the model hand (Botvinick and Cohen, 1998; Ehrsson et al., 2005). Notably, we do not rely on experimental measures in isolation; we always use them in combination with the questionnaires. Thus, according to our criteria, the confirmation of the rubber hand illusion would require both a significant proprioceptive drift toward the rubber hand and significant affirmative rating scores on the questionnaires (see Rohde et al., 2011 for further discussion of the risk of relying on proprioceptive drift measure alone).

With their eyes closed, participants used their left index finger to indicate the perceived position (height) of their right index finger. Participants were asked to make one rapid but accurate pointing movement by touching a board attached to the side of the model hand box (Figure 1B). To identify the position of each participant's left index finger, the board was covered with a sheet of paper with a millimeter grid, and the experimenter used a pen to mark the position corresponding to the central part of the participant's fingernail on this paper. The participants were asked to make one pointing movement before the illusion induction period and one pointing movement after this period, and the proprioceptive drift in the vertical plane was then calculated by subtracting the position of the first pointing from that of the second pointing (postpointing minus prepointing). Positive values indicated an upward drift in the subjects' sense of the hand's position, i.e., toward the model hand. Each condition was tested three times, and the average drift across the three trials was computed for each participant.

The order of the trials was pseudo-randomized and evenly distributed across the participants. The participants engaged in six trials in Experiment 2 and a total of 12 trials in Experiment 4. At the end of each experiment and after the pointing procedures, each condition was tested one more time ( $90 \mathrm{~s}$ per condition), and the participants were asked to complete a shorter version of the questionnaire with eight questions to measure their subjective experiences (see Table 1). The questionnaire data were collected to test for possible correlations between the subjectively experienced strength of ownership and proprioceptive drift (as reported for the classic rubber hand illusion by Botvinick and Cohen, 1998).

\section{STATISTICAL ANALYSIS OF QUESTIONNAIRE DATA}

All statistical comparisons were based on our a priori hypotheses unless explicitly mentioned otherwise in the text. In other words, these tests correspond to planned comparisons unless described as post hoc tests. The dependent variables were the measures of ownership and agency, as described in Section "Measures of the Illusion," i.e., the questionnaire ratings or the proprioceptive drift measure. First, the ownership and agency scores were separately compared with their respective control statements for each experimental condition to indicate whether a significant sense of ownership or agency occurred in each condition. Second, the ownership and agency statement scores were compared across conditions to test for significant differences, in accordance with our hypotheses (see Introduction). Correlation analyses (Spearman correlations) were also conducted to investigate possible systematic relationships between ownership and agency in the various experimental conditions. We evaluated the hypothesis that a correlation exists between the ownership statement score and the degree of proprioceptive drift in Experiment 2.

All data were assessed for a normal distribution using the Shapiro-Wilk test $(p>0.05)$, and the appropriate non-parametric tests were applied when one or more of the corresponding data sets failed to meet the criteria for normal distribution. The questionnaire data in Experiments 1 and 3 were not normally distributed; therefore, a Wilcoxon signed-rank test was used for pairwise comparisons. Multiple pairwise comparisons were conducted in Experiment 3; therefore, Bonferroni correction was applied. In Experiment 3, the Friedman test was also used to compare multiple conditions. In Experiment 4, we used repeated-measures ANOVA because the data were normally distributed.

All the tests were two-tailed. Asterisks in the plots indicate significance levels: ${ }^{*} p<0.05 ;{ }^{* *} p<0.01$; and ${ }^{* * *} p<0.001$. All analyses were conducted using the SPSS software package (version 19, SPSS Inc., Chicago, IL, USA).

\section{RESULTS}

\section{EXPERIMENT 1: QUESTIONNAIRE RESULTS FOR THE SYNCHRONOUS VERSUS ASYNCHRONOUS MOVEMENTS}

The questionnaire data clearly indicated that, at the group level, the participants experienced a strong illusion of ownership over the model hand and a strong sense of agency over its movements in the synchronous condition, with $75 \%$ of the participants affirming the illusion of ownership (i.e., an ownership score $\geq+1$ ). The mean ratings for ownership (1.63, $\mathrm{SD} \pm 1.27)$ and agency (2.44, $\mathrm{SD} \pm 0.56)$ were well above +1 in this condition. In contrast, the mean ratings were well below +1 in the asynchronous condition (ownership $=-0.99, \mathrm{SD} \pm 1.5$; agency $=0.66, \mathrm{SD} \pm 1.19$ ), demonstrating that the majority of participants denied experiencing ownership or agency in this control condition.

In the statistical analyses of the data, the ownership statement was first compared with the ownership-control statement, and the agency statement was compared with the agency-control statement, both for the synchronous condition (see Figure 2). The 


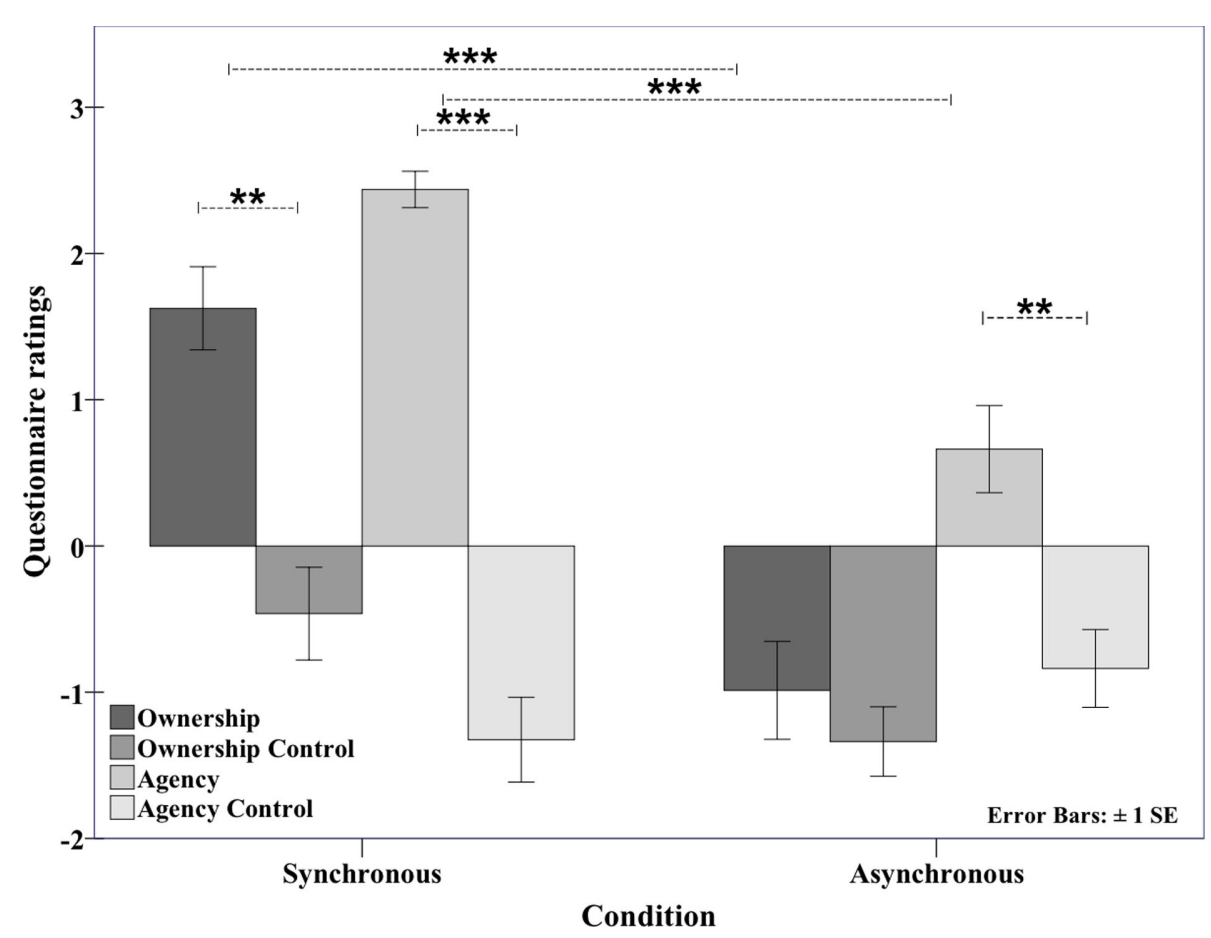

FIGURE 2 | Questionnaire data showing the average ownership and agency ratings on a 7-point Likert scale. The senses of ownership and agency were present (both $>1$ ) in the synchronous condition but not in the asynchronous condition. The ownership and agency statement scores (the means of the four statements related to ownership and agency, respectively) were significantly greater than their respective control statements in the synchronous condition. Additionally, both the ownership and agency statement scores were greater in the synchronous than asynchronous condition (see Experiment 1: Questionnaire results for the synchronous versus asynchronous movements). ${ }^{* *} p<.01 ;{ }^{* *} p<.001$. ownership and agency statements were then compared across conditions. The participants experienced a significant illusion of ownership in the synchronous condition (ownership versus ownership control, synchronous; $Z=3.792, p<0.001$ ), and this illusion was significantly stronger than in the asynchronous condition (ownership, synchronous versus asynchronous: $Z=3.304, p=0.001$ ). Similarly, the participants experienced a feeling of agency toward the model hand in the synchronous condition (agency versus agency control, synchronous; $Z=3.825, p<0.001$ ) that was significantly stronger than that in the asynchronous condition (agency synchronous versus asynchronous: $Z=3.767, p<0.001$ ).

\section{EXPERIMENT 2: PROPRIOCEPTIVE DRIFT DURING SYNCHRONOUS AND ASYNCHRONOUS ACTIVE MOVEMENTS}

After experiencing the synchronous condition, the participants' pointing responses indicated that they perceived their right hand to be closer to the model hand, which was above the box. A proprioceptive drift was present in the synchronous condition $(1.73 \mathrm{~cm}, \mathrm{SD} \pm 2.05)$ but not in the asynchronous condition $(-0.35 \mathrm{~cm}, \mathrm{SD} \pm 1.04)$, and importantly, the two conditions displayed a highly significant difference (Wilcoxon signed-rank test: $Z=3.062$, $p=0.002$; see Figure 3). These results complement the questionnaire data, and together, these data provide conclusive evidence that voluntary synchronous movements elicit the rubber hand illusion.

The observation that the degree of proprioceptive drift toward the model hand was significantly correlated with the questionnaire

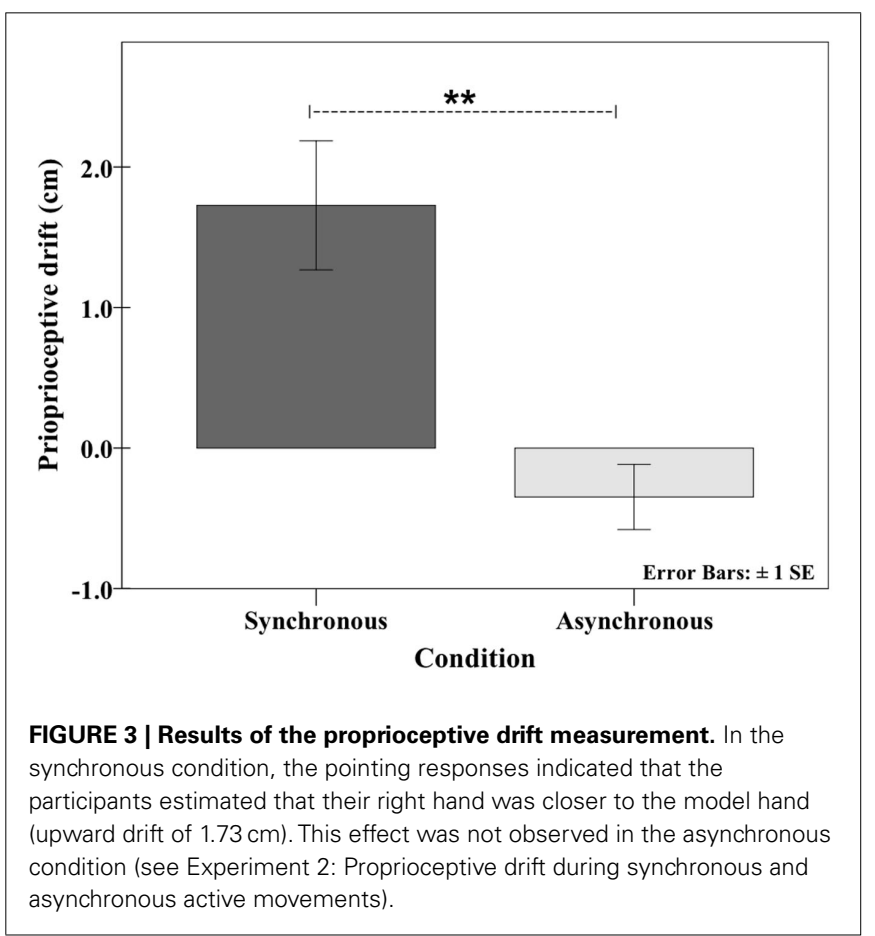

ownership statement ratings for the synchronous condition (Spearman $r=0.557, n=17, p=0.020$; see Figure 4) provides 

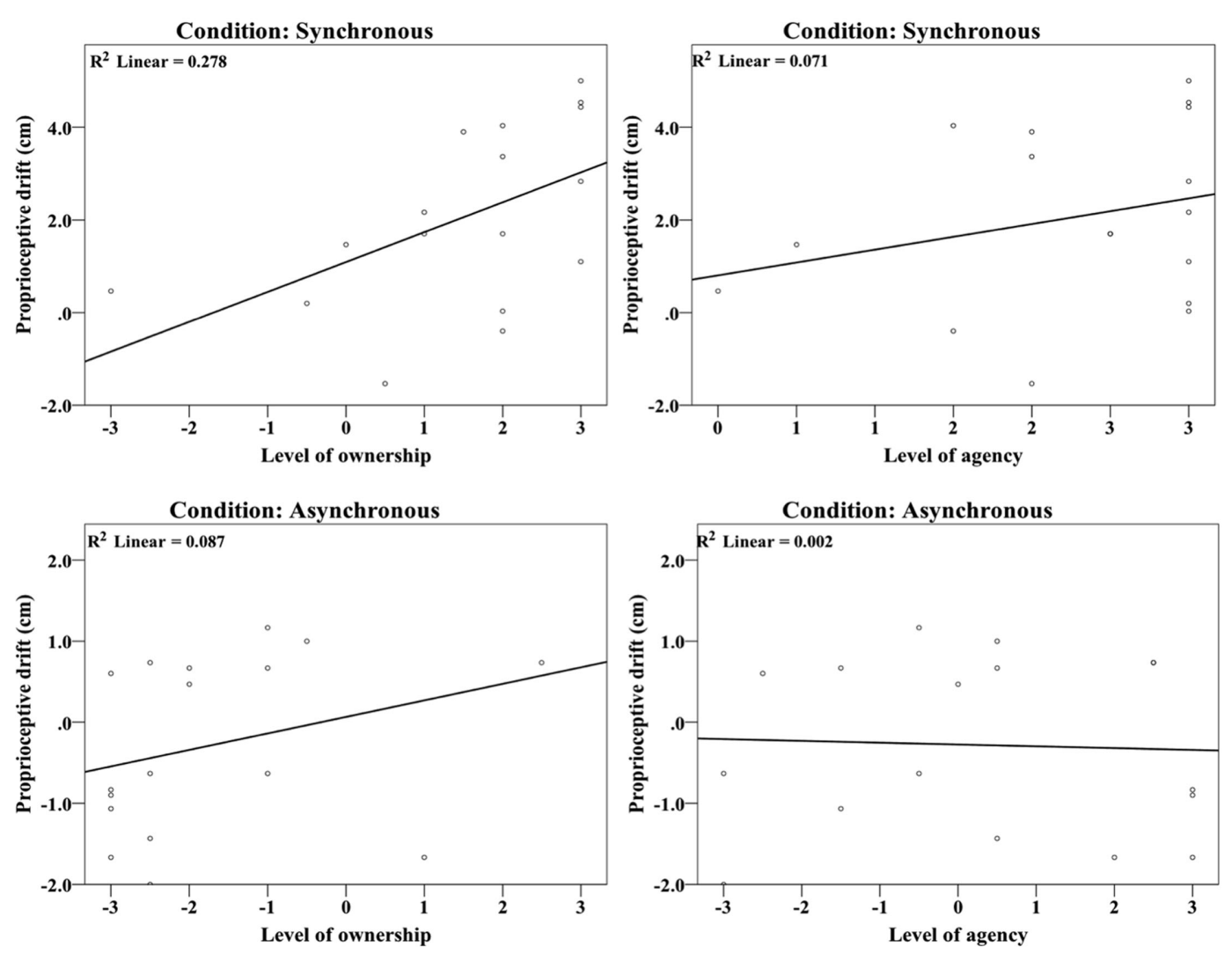

FIGURE 4 | Correlation analysis between proprioceptive drift and the ownership and agency ratings in the synchronous and asynchronous

conditions. As expected, the proprioceptive drift was correlated with the level of ownership $(p<0.05)$ but not with the level of agency (n.s.).

additional evidence for this claim (i.e., it reproduces Botvinick and Cohen's original observation). In the asynchronous condition, we only observed a trend toward significance (Spearman $r=0.457$, $n=17, p=0.065$ ). This significant correlation does not change when the outlier in the synchronous condition (level of ownership of -3 ) is removed (Spearman $r=0.532, n=16, p=0.034$ ) or when the data from both the synchronous and asynchronous conditions are pooled (Spearman $r=0.676, n=34, p<0.001$ ). No correlation was observed between proprioceptive drift and the agency statement in either of the two conditions (synchronous: Spearman $r=0.274, n=17, p=0.287$; asynchronous: Spearman $r=-0.020, n=17, p=0.938$ ). Thus, the proprioceptive drift is related to the experience of ownership but is not related to agency. Regrettably, questionnaire data sets from three participants were lost and hence could not be included in this analysis.

\section{EXPERIMENT 3: QUESTIONNAIRE DATA FOR THE ACTIVE/PASSIVE AND CONGRUENT/INCONGRUENT MOVEMENTS}

The questionnaire results clearly indicated the hypothesized pattern of results: participants rated the active congruent condition positively for both ownership (2.12, SD \pm 1.03$)$ and agency $(2.52, \mathrm{SD} \pm 0.55)$. In the active incongruent condition, agency $(2.2, \mathrm{SD} \pm 0.78)$ was affirmed, but ownership was not $(-0.62$, $\mathrm{SD} \pm 1.62)$. The opposite pattern was observed in the passive congruent condition, with affirmative ratings for ownership (1.57,
$\mathrm{SD} \pm 1.3)$ but not agency $(-0.61, \mathrm{SD} \pm 1.5)$. Finally, in the passive incongruent condition, the participants gave negative ratings for ownership $(-0.91, \mathrm{SD} \pm 1.6)$ and agency $(-1.5, \mathrm{SD} \pm 1.64)$ (see Figure 5). Thus, the hypothesized double dissociation of ownership and agency was observed at the qualitative level.

\section{Analysis of illusion versus control statements}

A quantitative statistical analysis was first performed to test whether the positively rated ownership and agency statements were significantly higher than their corresponding control statements within each condition. In the active congruent condition, both ownership and agency were significantly higher than the respective control categories (ownership versus ownership control: $Z=4.940, p<0.001$; agency versus agency control: $Z=4.939$, $p<0.001)$. In the passive congruent condition, the ownership statement was significantly higher than the ownership control statement $(Z=4.940, p<0.001)$. In the active incongruent condition, the agency statement was significantly higher than the agency control statement $(Z=4.920, p<0.001)$.

\section{Analysis of ownership across conditions}

Ownership statement scores differed significantly across the four conditions (Friedman test; $\chi^{2}[3, n=32]=65.287, p<0.001$ ). To test whether manipulating the orientation of the model hand produced the expected effect on ownership (i.e., higher ownership scores in the congruent conditions), ownership scores were 


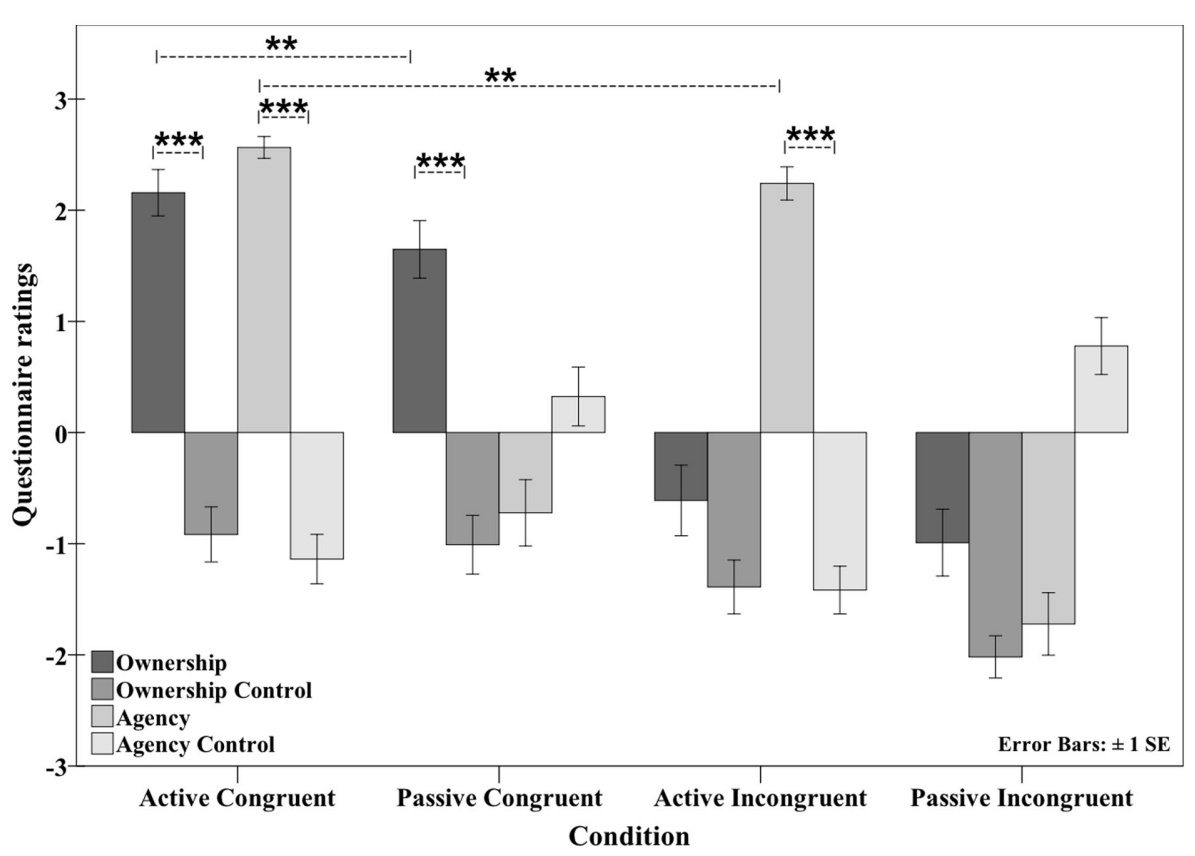

FIGURE 5 | Questionnaire data showing the mean ratings in the four conditions. The mode of movement (active versus passive) and the position (congruent versus incongruent) were varied. A sense of both ownership and agency was observed in the active congruent condition. In the passive congruent condition, participants experienced ownership only, whereas in the active incongruent condition, only agency was experienced. In the passive incongruent condition, neither ownership nor agency was experienced (see Experiment 3: Questionnaire data for the active/passive and congruent/incongruent movements). ${ }^{*} p<0.01$; ${ }^{* * *} p<0.001$. compared between the congruent conditions (active congruent and passive congruent) and the corresponding incongruent conditions (active incongruent and passive incongruent, respectively) to determine the main effect of position on ownership score. Because the data were not normally distributed, ANOVA was not possible. Importantly, ownership scores were higher in both congruent conditions than in the corresponding incongruent conditions (active congruent versus active incongruent: $Z=4.863, p<0.001$; passive congruent versus passive incongruent: $Z=4.656, p<0.001)$.

We then investigated whether the mode of movement modulated ownership scores (i.e., leading to higher ownership scores during active movements than passive movements). A significant difference was observed between the active congruent and passive congruent conditions $(Z=2.765, p<0.01)$ but not between the active incongruent and passive incongruent conditions $(Z=1.961, p=0.050)$ with the significance level set to $p<0.0125$ after Bonferroni correction. Therefore, when the rubber hand was in a congruent position, active movements increased ownership ratings, whereas in the incongruent position, this effect did not reach significance. To test for an interaction between the two factors (movement mode and position) across the four conditions, similar to an interaction effect of ANOVA, the differences in the individual ownership scores were calculated (active congruent minus active incongruent and passive congruent minus passive incongruent), and the mean values were compared. No significant difference was observed between the means $(t=0.744, \mathrm{~d} f=31$, $p=0.462$, two-tailed).

We should also briefly note here that we did not observe a significant difference in the objective measure of the rubber hand illusion between the active and passive conditions (see Experiment 4 and the proprioceptive drift measure), which together with the lack of interaction in the questionnaire data, argues against the notion that the participants experienced differences in the ownership illusion in the active and passive conditions.

\section{Analysis of agency across conditions}

Similarly, the agency statement varied significantly across the four conditions (Friedman test; $\chi^{2}[3, n=32]=75.247, p<0.001$ ). The first hypothesis we examined was whether the mode of movement (active or passive) had the expected effect on agency, i.e., higher agency scores inactive conditions than in passive conditions. Agency scores were compared between the active congruent and passive congruent conditions and between the active incongruent and passive incongruent conditions, and in both cases, the agency scores were higher in the active conditions (active congruent versus passive congruent: $Z=4.863, p<0.001$; active incongruent versus passive incongruent: $Z=4.772, p<0.001$ ).

The levels of agency were then compared between the congruent conditions (active congruent and passive congruent) and the corresponding incongruent conditions (active incongruent and passive incongruent) to explore the possible effect of hand position on agency (i.e., whether agency scores were higher in the congruent conditions than in the incongruent conditions). Here, the agency ratings were significantly higher in the congruent conditions than in the corresponding incongruent conditions (active congruent versus active incongruent: $Z=2.631, p<0.001$; PC versus PI: $Z=3.207, p=0.001)$, with the significance level set at $p<0.0125$ after Bonferroni correction. Notably, significant 
differences were found in both comparisons, even between the passive conditions (passive congruent versus passive incongruent) in which participants did not affirm experiencing agency per se.

To test for an interaction between the factors of movement mode and hand orientation, the differences in the individual agency scores were calculated (active congruent minus passive congruent and active incongruent minus passive incongruent) and the two mean values were compared. Here, using a two-tailed test, we found a nearly significant difference in the means $(t=-1.950$, $\mathrm{d} f=31, p=0.060$, two-tailed).

Together, these results indicate that the manipulation of the model hand position (congruent versus incongruent, for ownership) and the mode of movement (active versus passive, for agency) are the main factors that determine whether ownership and agency are experienced in the present design. Furthermore, subjects gave higher agency ratings when the arm was placed in an anatomically congruent position, irrespective of whether the movement was active or passive. The interaction approached significance, which suggests that the congruent position enhanced the level of agency specifically in the context of ownership.

\section{Analysis of the proportion of responders}

Finally, we reasoned that the observed ratings differences between the conditions may be rooted in either a) a general tendency to give lower ratings for one of the conditions (i.e., lower ratings but a similar proportion of responders) or b) different proportions of participants experiencing an illusion (i.e., fewer responders but similar levels of affirmation reported by participants who experienced an illusion). To investigate these possibilities, the ownership and agency statements were classified as either responders (positive rating $>1$ ) or non-responders $(\leq 0)$, and the proportion of responders for each condition was calculated and compared in a post hoc analysis. In the active congruent and passive congruent conditions, 87.5 and $78.1 \%$ of the participants, respectively, were classified as ownership responders. In the active congruent and active incongruent conditions, 96.9 and $93.8 \%$ of the participants, respectively, were classified as agency responders. A McNemar test revealed that there was no significant difference between the numbers of ownership responders in the active congruent and passive congruent conditions ( $n=32, p=0.375$ ) or between the numbers of agency responders in the active congruent and active incongruent conditions $(n=32, p=1.00)$. Thus, the equal proportions of responders in the two conditions that elicited ownership or agency indicate that the strength of these experiences among responders produced the significant differences in the mean ratings.

Unexpectedly, we observed a small increase in the ratings of the agency control statements in the passive congruent and passive incongruent conditions. However, these increases never exceeded +1 and were thus never clearly positively rated. Therefore, this observation does not affect any of the main comparisons or conclusions discussed above because, in agreement with our hypothesis, agency was inhibited in both conditions.

\section{Correlation between ownership and agency}

Interestingly, ownership and agency statements were significantly correlated in the active congruent condition (Spearman: $r=0.503$, $n=32, p=0.003$ ) but not in any of the other conditions (AI: $r=0.198, n=32, p=0.277$; PC: $r=0.220, n=32, p=0.226$; PI: $r=0.191, n=32, p=0.295$; see Figure 6). As illustrated by the plots in Figure 6, there was no relationship between ownership and agency ratings when the model hand was placed in an anatomically incongruent position.

\section{EXPERIMENT 4: PROPRIOCEPTIVE DRIFT FOR THE ACTIVE/PASSIVE AND CONGRUENT/INCONGRUENT MOVEMENTS}

In the proprioceptive drift pointing task, clear effects were observed in the active congruent $(+0.77 \mathrm{~cm})$ and passive congruent $(+0.6 \mathrm{~cm})$ conditions but not in the active incongruent $(-0.35 \mathrm{~cm})$ and passive incongruent $(-0.004 \mathrm{~cm})$ conditions. Repeated-measures $2 \times 2$ ANOVA revealed a significant effect of position $(F[1,31]=7.878, p<0.01)$ but not mode of movement generation $(F[1,31]=0.144, p=0.707)$ and no significant interaction between the two (see Figure 7). Thus, similar levels of ownership, represented by the proprioceptive drift measure, were observed when the model hand was presented in an anatomically congruent condition, regardless of whether the movements were active or passive.

\section{COMPARING THE EFFECTS OF ASYNCHRONY AND PASSIVE MOVEMENT ON AGENCY ACROSS EXPERIMENTS}

The experiments described above demonstrated that both visuosomatic asynchrony and passive movement significantly reduced the sense of agency. Determining which experimental manipulation was the most effective at eliminating agency had some bearing on the underlying processes involved (see Discussion). Therefore, the agency statement ratings were directly compared in a post hoc test between the asynchronous condition (group mean score 0.66 in Experiment 1) and the passive congruent condition (group mean score -0.61 in Experiment 3 ), using an unpaired $t$ test. This analysis revealed that the agency ratings in the passive condition were significantly weaker than those in the asynchronous condition $(t=3.105, \mathrm{~d} f=50, p=0.003$, two-tailed), demonstrating that passive movement eliminated agency more completely than visuo-somatic asynchrony.

\section{DISCUSSION}

This study yielded three main findings. First, the participants could experience a physical, moving model hand as their own hand, just as illusory ownership is experienced in the traditional rubber hand illusion. Second, our results demonstrate that the feelings of ownership and agency can be dissociated, suggesting that these sensations represent independent processes of the human brain. A mismatch between the observed position of the model and the sensed position of the real hand (anatomical incongruence) eliminates ownership but does not disrupt agency. Passive movement, in contrast, eliminates agency but leaves ownership intact. As expected, both processes require temporal congruence between the observed movement of the model index finger and the actual movement of the hidden index finger. Third, we found that ownership modulated agency, and thus, stronger agency was experienced when the model hand was perceived to be a part of one's body. Moreover, we only observed a significant correlation between the ownership and agency ratings in the conditions where the model was owned. In the following sections, we discuss how these findings impact cognitive neuroscience research on ownership and agency. 

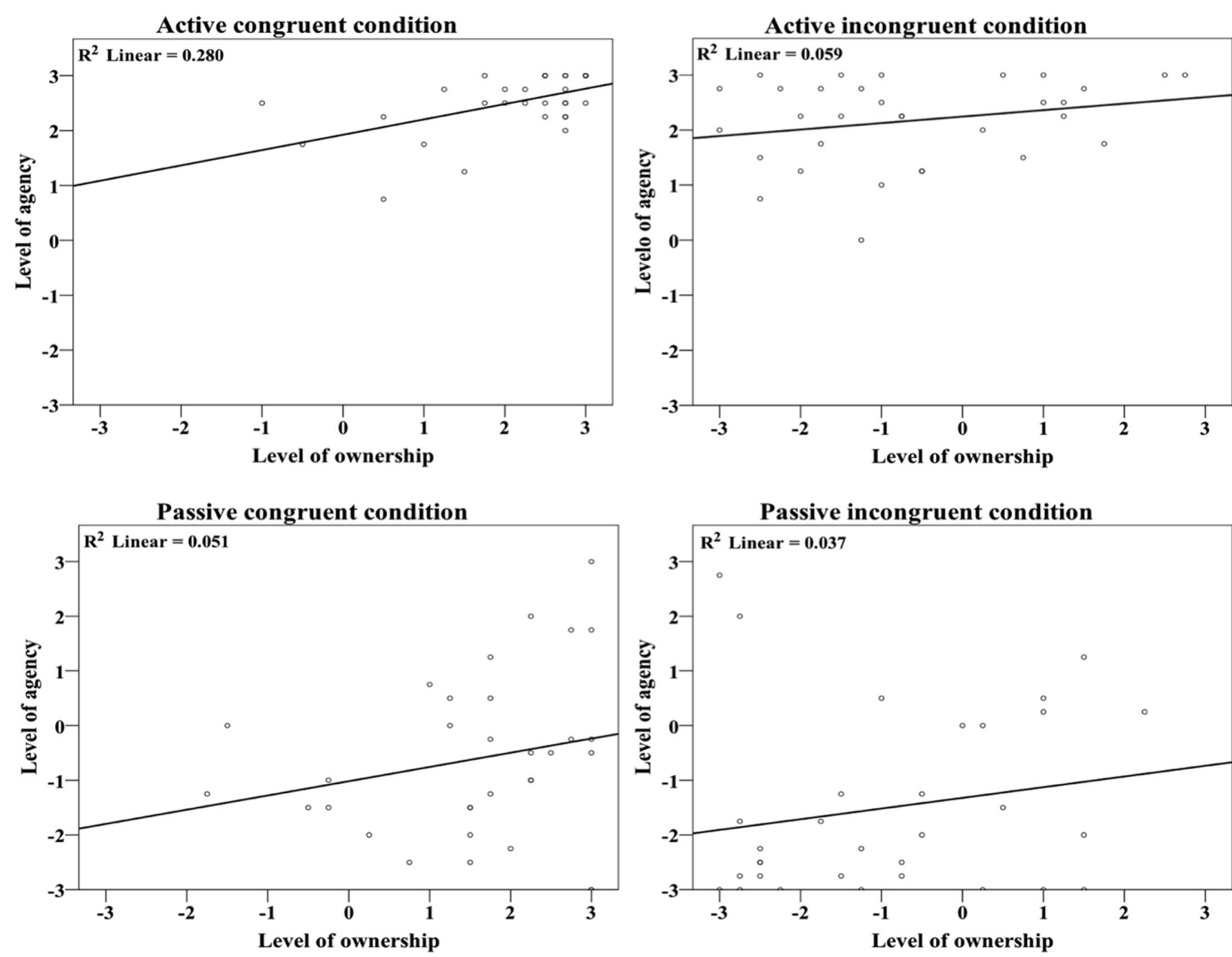

FIGURE 6 | Plots showing the correlations between the ownership and agency statement scores. These scores were significantly correlated only in the active congruent condition ( $r=0.503, p=0.003)$

\section{THE SENSE OF OWNERSHIP}

The present results clearly show that correlated finger movements of a model hand and a participant's hand can elicit an illusion of ownership over the moving model hand in the absence of tactile stimulation by a brush or other external object, as in the classic rubber hand illusion. We observed strong affirmative ratings and significant proprioceptive drift in the execution of both active and passive movements as well as significant correlations between proprioceptive drift and the strength of ownership, as reported in questionnaire ratings. In our experience, the ownership illusion takes time to develop, approximately 10-30 s (anecdotal observations), which is consistent with the classic rubber hand illusion (Ehrsson et al., 2004; Lloyd, 2007). These observations reproduce key observations from studies investigating the classic rubber hand illusion elicited by applying brush strokes to the two hands (Botvinick and Cohen, 1998; Ehrsson et al., 2004; Tsakiris and Haggard, 2005). However, in the present moving rubber hand paradigm, the sense of ownership results from a match between spatially and temporally congruent somatic and visual information from the moving rubber finger and the participant's finger. Multisensory brain areas, such as the ventral premotor cortex, intraparietal cortex, putamen, and lateral cerebellum, likely compare these signals (Graziano and Gross, 1993; Graziano, 1999; Graziano et al., 2000; Hagura et al., 2007; Gentile et al., 2011). Extending the findings of the classic rubber hand illusion, the present study demonstrates that meaningful correlations among all available sensory channels are critical for body ownership rather than signals from a specific sensory modality, such as vision or a particular type of peripheral somatosensory receptor. This interpretation is consistent with the finding that the rubber hand illusion can be elicited in blindfolded participants (by having them touch a rubber right hand with their left index finger in synchrony with touches applied to their own right hand; Ehrsson et al., 2005) or when the cutaneous information is eliminated by anesthetizing the digital nerves (Walsh et al., 2011).

\section{THE SENSE OF AGENCY}

The participants in our study reported a strong sense of agency over the movements of the model hand in the active (synchronous) conditions, regardless of whether the hand was placed in an anatomically plausible position. Thus, agency was also reported when the rubber hand was experienced as an external object, consistent with our hypothesis that agency can be extended to object-directed actions and tool use (see Introduction). Our results also demonstrate that both asynchronous and passive movement reduces agency. Thus, the generation of agency requires both motor intention and a match between the predicted sensory feedback and the actual sensory feedback of the movements. Interestingly, we observed significantly different effects of these manipulations across the experiments. During passive movements 


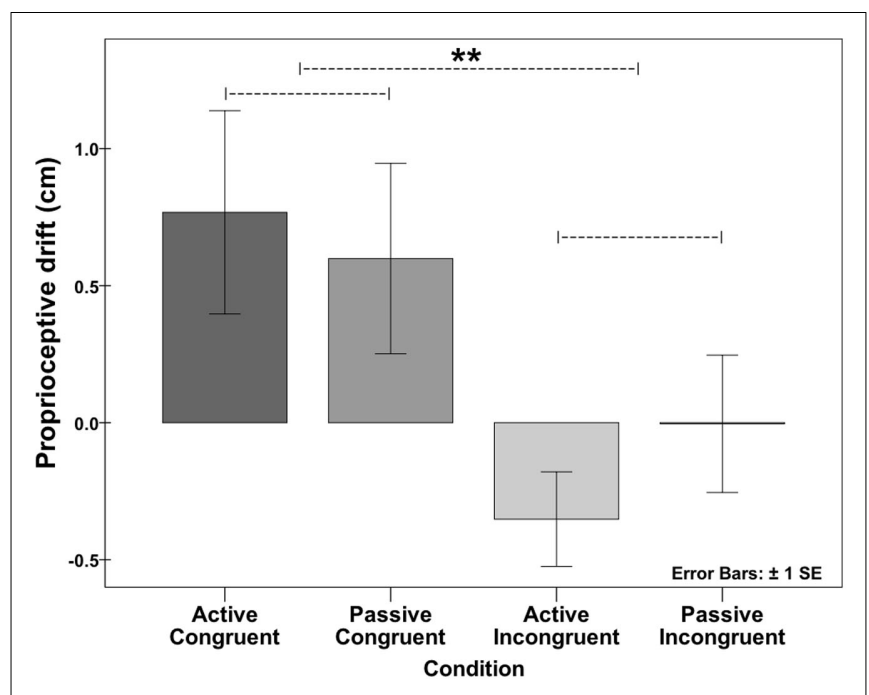

FIGURE 7 | Proprioceptive drift in the four conditions of Experiment 3. A significant proprioceptive drift in the perceived location of the right hand toward the model hand was observed in the two conditions in which the model hand was placed in the anatomically congruent position to elicit the ownership illusion (effect of position $(F[1,31]=7.878, p<0.01)$, but the mode of movement did not have a significant effect (effect of mode $(F[1,31]=0.144, p=0.707)$; see Experiment 4: Proprioceptive drift for the active-passive and congruent-incongruent movements).

(Experiment 3), the sense of agency broke down more completely than in the asynchronous manipulation, in which a slightly positive level of agency remained (Experiment 1; see Comparing the effects of asynchrony and passive movement on agency across experiments). This finding indicates that as long as the intention to move is present, there exists an inherent tendency to attribute authorship of actions to the self, which is then cancelled by the detection of mismatches between predicted sensory feedback and actual sensory feedback. Without the intention to move (passive movements), agency is more completely abolished because without motor intention, no efference copy signals are generated from the motor areas, and therefore, presumably no sensory consequences of movement are computed (or they are predicted to be zero). These conclusions are in agreement with those of an earlier study by Farrer et al. (2008b), in which participants were exposed to temporal and spatial deviations of their own movements and were asked to judge whether the perceived movement was generated by "me," by an "other," or by "me" but "biased." During the temporal delays, the participants predominantly judged their actions as being generated by themselves or as "biased" but not as generated by an "other." Thus, when the intention of action was present, participants demonstrated a tendency to consider this feedback or effect to be self-generated although it appeared delayed. Therefore, the sense of agency is best explained by a model in which both motor intentions and comparator mechanisms are essential contributing factors.

However, a question remains: do the senses of agency over external objects and one's body parts truly represent identical neuro-cognitive processes? It can be argued that the agency experienced when the model hand was rotated was akin to the feeling of causation elicited by moving a computer mouse on a screen or using a tool (external agency). When the participants simultaneously felt agency and ownership over the model hand in a congruent position, the experience of agency may be more similar to that of voluntarily raising one's arm (body agency). We did not explicitly design the questionnaire statements to distinguish possible phenomenological differences between the agency experiences in these two situations. However, informal interviews with the naïve participants after the experiments at least suggest that during synchronous movements of a congruently placed hand, the participant genuinely experienced agency of the model hand "exactly like when I move my real hand.” Three specific observations suggest that body agency may contribute an additional component over and above the basic agency experienced when controlling an external object. First, the participants reported significantly stronger agency in the active congruent condition than in the active incongruent condition $(p<0.001)$, i.e., stronger agency when the model hand was perceived as part of one's body than when it was perceived to be an external object. Additional support for this claim was the observation that a (non-parametric) test for the interaction between arm orientation (congruent versus incongruent) and the mode of movement (active versus passive) almost reached significance ( $p=0.06$, two-tailed; see Experiment 3: Questionnaire data for the active/passive and congruent/incongruent movements for details). In other words, the participants experienced the greatest agency when the model hand was placed in an anatomically congruent position and the finger movements were actively generated. Second, we observed a significant correlation between agency and ownership ratings but only in the active congruent condition, in which participants also experienced ownership of the model hand. This finding indicates that the experience of body agency is more closely linked to the feeling of body ownership than to external agency. Third, we noted stronger affirmation of ownership in the active conditions than in the passive conditions, a difference that reached significance when the hand was presented in an anatomically congruent position $(p<0.0125$ after correction for multiple comparisons) but not in the incongruent position. This result indicates that body agency may exhibit a stronger facilitatory effect on ownership than external agency. Taken together, these three observations provide indirect evidence that body agency is distinct from agency over external objects. Clearly, an important goal for future behavioral and brain-imaging studies will be to provide objective evidence for this hypothesis.

What specific mechanisms might support body agency? When experiencing agency over one's body, the executed movement is aligned with the preceding motor intention, and a match occurs between the corresponding predicted and actual visuo-somatic feedback from the limb in question. These sensory predictions are fine-tuned to the spatial and temporal characteristics of the human skeletomuscular apparatus (Bays et al., 2005). This scenario presumably implies that body agency is sensitive to the spatial congruency of intended and executed movements in bodypart-centered reference frames (Graziano et al., 1997), similar to the spatial constraint of limb ownership (Lloyd, 2007; Makin et al., 2008). Thus, we propose that feelings potentially specifically associated with body agency, i.e., the illusory sensation that the model hand can be moved voluntarily just like the real hand, are related 
to the transfer of these sensorimotor integration mechanisms to the model hand.

In contrast, agency over external objects is not constrained by the skeletomuscular characteristics and spatial reference frames of the human body. Instead, this type of agency relies on sensory predictions based on learned arbitrary mappings between an action and a goal (Prinz, 2003; Spengler et al., 2009). There is evidence that once this mapping is established and the action is linked to a specific goal, the particular movement used to achieve this goal is no longer of primary importance and that the action is coded in more general goal-specific terms (Prinz, 2003). Thus, participants can experience agency over external sensory events that match the predicted goal of the self-generated actions, even when these clearly violate basic anatomically plausible sensorimotor predictions (Preston and Newport, 2010).

In addition to these specific effects, we observed higher agency ratings in all anatomically congruent conditions that elicited illusory ownership, including passive conditions in which subjects generally denied agency. Thus, it appears that even in the absence of an engaged agency mechanism, the presence of ownership still drives a residual sense of agency. Consistent with this idea, Longo et al. (2008) observed that during the traditional (static) rubber hand illusion, participants experienced a specific feeling of control over the rubber hand, although the change in questionnaire scores was relatively low. Intuitively, it seems plausible that an entity that is experienced as a part of one's body may be associated with at least some sense of agency. Indeed, a common anecdotal observation from the classic rubber hand illusion is that when a person who is experiencing the illusion is asked to raise his or her index finger, they are surprised to find that the rubber hand does not move, as if they had expected to be able to control it. These observations may reflect a general tendency to ascribe agency to an owned body part, which is in agreement with previous reports of interactions between ownership and (body) agency (Tsakiris et al., 2007b, 2010).

\section{DOES AGENCY MODULATE OWNERSHIP?}

Previous researchers have suggested the possibility that agency supports the recognition of hand movements (van den Bos and Jeannerod, 2002; Tsakiris et al., 2005; Shimada et al., 2009a), and Dummer et al. (2009) observed higher ownership questionnaire ratings for active movements than for passive movements. Our data do not provide conclusive evidence that agency enhances ownership. First, the proprioceptive drift did not differ significantly between the active and passive movements (consistent with Tsakiris et al., 2006). This negative finding is crucial because our "golden standard" for measuring body ownership is the use of a combination of questionnaires and an objective measure, as relying on one approach alone may yield unreliable results (Rohde et al., 2011). Nevertheless, we noted that the participants gave higher affirmative ownership scores during active movements $($ mean score +2.12$)$ than during passive movements $(+1.57$; see Experiment 3: Questionnaire data for the active/passive and congruent/incongruent movements and Figure 5). However, this difference may not be specific to agency, as we found no significant interaction effect between movement type and the orientation of the arm (Experiment 3). Thus, we cannot rule out the possibility of non-specific effects related to general differences in task demands, either when performing active finger tapping or only relaxing the hand, as an explanation for the slightly higher ratings in the active conditions.

\section{POSSIBLE NEURAL SUBSTRATES}

The present behavioral results are consistent with the neurophysiological and neuroimaging literature in which the sense of agency and the feeling of body ownership have been associated with separate brain systems. Human electrophysiological, imaging, and neurodisruptive studies indicate that the pre-supplementary motor area may be involved in generating the conscious experience of motor intentions (Haggard, 2005; Lau et al., 2004, 2007). Furthermore, functional magnetic resonance imaging studies suggest that cerebellar regions and the right inferior parietal cortex may be key structures for implementing the comparisons of predicted and actual sensory feedback required for explicit agency judgments (Blakemore et al., 2001; Farrer and Frith, 2002; Farrer et al., 2003, 2008a; Blakemore and Sirigu, 2003; Tsakiris et al., 2010). In contrast, body ownership has been associated with activation in the ventral premotor cortex (Ehrsson et al., 2004, 2005; Petkova et al., 2011), the intraparietal cortex (Ehrsson et al., 2004, 2005; Petkova et al., 2011), and the putamen (Petkova et al., 2011), which are well-known cortical nodes for integrating visual, tactile, and proprioceptive information from the body and the space immediately surrounding the body in body-part-centered coordinates (Graziano and Gross, 1993; Fogassi et al., 1996; Graziano et al., 1997; Duhamel et al., 1998; Graziano, 1999). Thus, our present behavioral results that ownership and agency can be independently manipulated within a single rubber hand illusion paradigm may reflect the different brain circuits dedicated to these processes. However, given that both ownership and agency appear to involve anatomically interconnected nodes within the sensorimotor brain systems that control bodily action, additional imaging studies will be necessary to demonstrate a double dissociation between ownership and agency in the context of activation maps within the same group of subjects. Another candidate region is the posterior insula, which has been associated with both the sense of agency (Farrer and Frith, 2002; Farrer et al., 2003) and ownership based on an observed correlation between the proprioceptive drift and regional cerebral blood flow in a rubber hand illusion paradigm (Tsakiris et al., 2007a). Finally, future imaging studies should also test the hypothesis of differences in the neural bases of body agency and external agency.

\section{CONCLUSION}

In this study, we investigated the sense of ownership, the sense of agency, and their potential interactions in a single experimental setting where only one factor at a time was changed in otherwise equivalent conditions. A match between the observed orientation of the model and the sensed position of the hidden hand was required for ownership (anatomically congruent position), active movement was required for agency, and visuo-somatic synchrony between the observed and sensed actions of the real and model fingers were required for both agency and ownership. Together, these findings provide the first direct demonstration of the double dissociation of ownership and agency in a single rubber hand 
illusion paradigm. Furthermore, a specific interaction between the two processes was observed in that agency over an owned limb was reported as being felt more strongly than agency over external objects. These results advance our understanding of the critical factors involved in mediating ownership and agency and have implications for research in neuroprosthetics, virtual reality, and cognitive schizophrenia.

\section{AUTHOR CONTRIBUTIONS}

Andreas Kalckert and H. Henrik Ehrsson designed the experiments. Andreas Kalckert conducted the experiments and

\section{REFERENCES}

Bays, P. M., Wolpert, D. M., and Flanagan, J. R. (2005). Perception of the consequences of self-action is temporally tuned and event driven. Curr. Biol. 15, 1125-1128.

Blakemore, S.-J., and Frith, C. (2003). Self-awareness and action. Curr. Opin. Neurobiol. 13, 219-224.

Blakemore, S.-J., Frith, C., and Wolpert, D. M. (2001). The cerebellum is involved in predicting the sensory consequences of action. Neuroreport 12, 1879-1884.

Blakemore, S.-J., and Sirigu, A. (2003). Action prediction in the cerebellum and in the parietal lobe. Exp. Brain Res. 153, 239-245.

Botvinick, M. (2004). Neuroscience. Probing the neural basis of body ownership. Science 305, 782-783.

Botvinick, M., and Cohen, J. (1998). Rubber hands 'feel' touch that eyes see. Nature 391, 756 .

Chiel, H. J., and Beer, R. D. (1997). The brain has a body: adaptive behavior emerges from interactions of nervous system, body and environment. Trends Neurosci. 20, 553-537.

Churchland, P. (2002). Selfrepresentation in nervous systems. Science 296, 308-310.

Crapse, T. B., and Sommer, M. A. (2008). Corollary discharge across the animal kingdom. Nat. Rev. Neurosci. 9, 587-600.

David, N., Newen, A., and Vogeley, K. (2008). The "sense of agency" and its underlying cognitive and neural mechanisms. Conscious. Cogn. 17, 523-534.

Duhamel, J. R., Colby, C. L., and Goldberg, M. E. (1998). Ventral intraparietal area of the macaque: congruent visual and somatic response properties. J. Neurophysiol. 79, 126-136.

Dummer, T., Picot-Annand, A., Neal, T., and Moore, C. (2009). Movement and the rubber hand illusion. Perception 38, 271-280.

Ehrsson, H. H. (2012). "The concept of body ownership and its relation to multisensory integration," In
The New Handbook of Multisensory Processes, Chapter 43, ed. B. E. Stein (Cambridge, MA: MIT Press), 775-792.

Ehrsson, H. H., Holmes, N. P., and Passingham, R. E. (2005). Touching a rubber hand: feeling of body ownership is associated with activity in multisensory brain areas. J. Neurosci. 25, 10564-10573.

Ehrsson, H. H., Spence, C., and Passingham, R. E. (2004). That's my hand! Activity in premotor cortex reflects feeling of ownership of a limb. Science 305, 875-877.

Farrer, C., Franck, N., Georgieff, N., Frith, C., Decety, J., and Jeannerod, M. (2003). Modulating the experience of agency: a positron emission tomography study. Neuroimage 18, 324-333.

Farrer, C., Frey, S. H., van Horn, J. D., Tunik, E., Turk, D., Inati, S., and Grafton, S. T. (2008a). The angular gyrus computes action awareness representations. Cereb. Cortex 18, 254-261.

Farrer, C., Jeannerod, M., and Franck, N. (2008b). Effect of distorted visual feedback on the sense of agency. Behav. Neurol. 19, 53-57. riencing oneself vs. another person as being the cause of an action: the neural correlates of the experience of agency. Neuroimage 15, 596-603.

Fogassi, L., Gallese, V., Fadiga, L., Luppino, G., Matelli, M., and Rizzolatti, G. (1996). Coding of peripersonal space in inferior premotor cortex (area F4). J. Neurophysiol. 76, 141-157.

Franck, N., Farrer, C., Georgieff, N., Marie-Cardine; M., Daléry, J., d'Amato, T., and Jeannerod, M. (2001). Defective recognition of one's own actions in patients with schizophrenia. Am J Psychiatry 158, 454-459.

Frith, C., Blakemore, S.-J., and Wolpert, D. M. (2000). Abnormalities in the awareness and control of action. Philos. Trans. R. Soc. Lond. B Biol. Sci. 355, 1771-1788.
Farrer, C., and Frith, C. D. (2002). Expe-

analyzed the data. Andreas Kalckert and H. Henrik Ehrsson discussed the results and wrote the manuscript.

\section{ACKNOWLEDGMENTS}

This study was funded by the European Research Council (erc.europa.eu), the Swedish Foundation for Strategic Research (www.stratresearch.se/en), the Human Frontier Science Program (www.hfsp.org), Söderbergska Stiftelsen, and the Stockholm Brain Institute (www.stockholmbrain.se). The authors thank all the participants for their participation in this study.

Gallagher, S. (2000). Philosophical conceptions of the self: implications for cognitive science. Trends Cogn. Sci. 4 14-21.

Gallagher, S. (2005). How the Body Shapes the Mind. Oxford: Oxford University Press.

Gallagher, S. (2007). The natural philosophy of agency. Philos. Comp. 2, 347-357.

Gentile, G., Petkova, V., and Ehrsson, H. H. (2011). Integration of visual and tactile signals from the hand in the human brain: an FMRI study. J. Neurophysiol. 105, 910-922.

Graziano, M. S. (1999). Where is my arm? The relative role of vision and proprioception in the neuronal representation of limb position. Proc. Natl. Acad. Sci. USA 96, 10418-10421.

Graziano, M. S., and Botvinick, M. (2002). "How the brain represents the body: insights from neurophysiology and psychology," in Common Mechanisms in Perception and Action: Attention and Performance XIX, eds W. Prinz and B. Hommel (Oxford: Oxford University Press), 136-157.

Graziano, M. S., and Cooke, D. F. (2006). Parieto-frontal interactions, personal space, and defensive behavior. Neuropsychologia 44, 845-859.

Graziano, M. S., Cooke, D. F., and Taylor, C. S. (2000). Coding the location of the arm by sight. Science 290, 1782-1786.

Graziano, M. S., and Gross, C. G. (1993). A bimodal map of space: somatosensory receptive fields in the macaque putamen with corresponding visual receptive fields. Exp. Brain Res. 97, 96-109.

Graziano, M. S., Hu, X. T., and Gross, C. G. (1997). Visuospatial properties of ventral premotor cortex. J. Neurophysiol. 77, 2268-2292.

Haggard, P. (2005). Conscious intention and motor cognition. Trends Cogn. Sci. 9, 290-295.

Haggard, P., Clark, S., and Kalogeras, J. (2002). Voluntary action and conscious awareness. Nat. Neurosci. 5, 382-385.
Hagura, N., Takei, T., Hirose, S., Aramaki, Y., Matsumura, M., Sadato, N., and Naito, E. (2007). Activity in the posterior parietal cortex mediates visual dominance over kinesthesia. $J$. Neurosci. 27, 7047-7053.

Holmes, N. P., and Spence, C. (2005). Multisensory integration: space, time and superadditivity. Curr. Biol. 15, R762-R764.

Ijsselsteijn, W. A., de Kort, Y. A. W., and Haans, A. (2005). Is this my hand i see before me? The rubber hand illusion in reality, virtual reality, and mixed reality. Presence, Teleop. Viot. 15, 455-464.

Imamizu, H., Miyauchi, S., Tamada, T., Sasaki, Y., Takino, R., Putz, B., Yoshioka, T., and Kawato, M. (2000). Human cerebellar activity reflecting an acquired internal model of a new tool. Nature, 403, 192-195.

Jeannerod, M. (2003). The mechanism of self-recognition in humans. Behav. Brain Res. 142, 1-15.

Kammers, M., Longo, M. R., Tsakiris, M., Dijkerman, H. C., and Haggard, P. (2009). Specificity and coherence of body representations. Perception 38, 1804-1820.

Lau, H. C., Rogers, R. D., Haggard, P., and Passingham, R. E. (2004). Attention to intention. Science 303 , 1208-1210.

Lau, H. C., Rogers, R. D., and Passingham, R. E. (2007). Manipulating the experienced onset of intention after action execution. J. Cogn. Neurosci. 19, 81-90.

Lloyd, D. M. (2007). Spatial limits on referred touch to an alien limb may reflect boundaries of visuotactile peripersonal space surrounding the hand. Brain Cognit. 64, 104-109.

Longo, M. R., and Haggard, P. (2009). Sense of agency primes manual motor responses. Perception 38, 69-78.

Longo, M. R., Schüür, F., Kammers, M. P. M., Tsakiris, M., and Haggard, P. (2008). What is embodiment? A psychometric approach. Cognition 107, 978-998. 
Makin, T., Holmes, N. P., and Ehrsson, H. H. (2008). On the other hand: dummy hands and peripersonal space. Behav. Brain Res. 191, $1-10$.

Merlau-Ponty, M. (1945). Phenomenology of Perception. London: Routledge Classics.

Newport, R., Pearce, R., and Preston, C. (2009). Fake hands in action: embodiment and control of supernumerary limbs. Exp. Brain Res. 204, 385-395.

Newport, R., and Preston, C. (2010). Pulling the finger off disrupts agency, embodiment and peripersonal space. Perception 39, 1296-1298.

Petkova, V., and Ehrsson, H. H. (2009). When right feels left: referral of touch and ownership between the hands. PLoS ONE 4, 6933.

Petkova, V. I., Björnsdotter, M., Gentile, G., Jonsson, T., Li, T. Q., and Ehrsson, H. H. (2011). From part to wholebody ownership in the multisensory brain. Curr. Biol. 21, 1118-1122.

Petkova, V. I., and Ehrsson, H. H. (2008). If I were you: perceptual illusion of body swapping. PLoS ONE, 3, e3832.

Preston, C., and Newport, R. (2010). Self-denial and the role of intentions in the attribution of agency. Conscious. Cognit. 19, 986-998.

Prinz, W. (2003). "Experimental approaches to action," In Agency and Self-awareness, eds J. Roessler and N. Eilan (Oxford: Oxford University Press), 165-187.

Rohde, M., Di Luca, M., and Ernst, M. O. (2011). The rubber hand illusion: feeling of ownership and proprioceptive drift do not go hand in hand. PLoS ONE 6, e21659.

Sanchez-Vives, M. V., Spanlang, B., Frisoli, A., Bergamasco, M., and Slater, M. (2010). Virtual hand illusion induced by visuomotor correlations. PLoS ONE 5, e10381.

Sato, A. (2009). Both motor prediction and conceptual congruency between preview and action-effect contribute to explicit judgment of agency. Cognition 110, 74-83.

Sato, A., and Yasuda, A. (2005). Illusion of sense of self-agency: discrepancy between the predicted and actual sensory consequences of actions modulates the sense of self-agency, but not the sense of self-ownership. Cognition 94, 241-255.

Shergill, S. S., Samson, G., Bays, P. M., Frith, C. D., and Wolpert, D. M. (2005). Evidence for sensory prediction deficits in schizophrenia. Am. J. Psychiatry 162, 2384-2386.

Shimada, S., Qi, Y., and Hiraki, K. (2009a). Detection of visual feedback delay in active and passive selfbody movements. Exp. Brain Res. 201, 359-364.

Shimada, S., Fukuda, K., and Kazuo, H. (2009b). Rubber hand illusion under delayed visual feedback. PLoS ONE 4, 6185 .

Slater, M., Perez-Marcos, D., Ehrsson, H. H., and Sanchez-Vives, M. V. (2008). Towards a digital body: the virtual arm illusion. Front. Hum. Neurosci. 2, 6 .

Slater, M., Perez-Marcos, D., Ehrsson, H. H., and Sanchez-Vives, M. V. (2009). Inducing illusory ownership of a virtual body. Front. Neurosci. 3 , 214-220.

Spengler, S., Cramon, von, D. Y., and Brass, M. (2009). Was it me or was it you? How the sense of agency originates from ideomotor learning revealed by fMRI. Neuroimage, 46, 290-298.

Stein, B. E., and Stanford, T. R. (2008). Multisensory integration: current issues from the perspective of the single neuron. Nat. Rev. Neurosci. 9, 255-266.

Synofzik, M., Vosgerau, G., and Newen, A. (2008a). Beyond the comparator model: a multifactorial two-step account of agency. Conscious. Cognit. 17, 219-239.
Synofzik, M., Vosgerau, G., and Newen, A. (2008b). I move, therefore I am: a new theoretical framework to investigate agency and ownership. Conscious. Cognit. 17, 411-424.

Tsakiris, M., and Haggard, P. (2005). The rubber hand illusion revisited: visuotactile integration and selfattribution. J. Exp. Psychol. Hum. Percept. Perform. 31, 80-91.

Tsakiris, M., Haggard, P., Franck, N. Mainy, N., and Sirigu, A. (2005). A specific role for efferent information in self-recognition. Cognition 96, 215-231.

Tsakiris, M., Prabhu, G., and Haggard, P. (2006). Having a body versus moving your body: how agency structures body-ownership. Conscious. Cognit. 15, 423-432.

Tsakiris, M., Hesse, M. D., Boy, C., Haggard, P., and Fink, G. R. (2007a) Neural signatures of body ownership: a sensory network for bodily self-consciousness. Cereb. Cortex 17 2235-2244.

Tsakiris, M., Schütz-Bosbach, S., and Gallagher, S. (2007b). On agency and body-ownership: phenomenological and neurocognitive reflections. Conscious. Cognit. 16 , 645-660.

Tsakiris, M., Longo, M. R., and Haggard, P. (2010). Having a body versus moving your body: neural signatures of agency and body-ownership. Neuropsychologia 48, 2740-2749.

van den Bos, E., and Jeannerod, $M$ (2002). Sense of body and sense of action both contribute to selfrecognition. Cognition 85, 177-187. von Holst, E., and Mittelstaedt, $\mathrm{H}$. (1950). Das Reafferenzprinzip WechselwirkungenzwischenZentralnervensystem und Peripherie. Die Naturwissenschaften 20 464-476.

Voss, M., Moore, J., Hauser, M., Gallinat, J., Heinz, A., and Haggrd, P. (2010). Altered awareness of action in schizophrenia: a specific deficit in predicting action consequences. Brain 133, 3104-3112.

Walsh, L. D., Moseley, G. L., Taylor, J. L., and Gandevia, S. C. (2011). Proprioceptive signals contribute to the sense of body ownership. J. Physiol 589, 3009-3012.

Wegner, D. M., Sparrow, B., and Winerman, L. (2004). Vicarious agency: experiencing control over the movements of others. J. Pers. Soc. Psychol. 86, 838-848.

Wittgenstein, L. (1958). Philosophical Investigations. Oxford: Blackwell.

Wolpert, D. M., Diedrichsen, J., and Flanagan, J. R. (2011). Principles of sensorimotor learning. Nat. Rev. Neurosci. 12, 739-751.

Wolpert, D. M., and Ghahramani, Z. (2000). Computational principles of movement neuroscience. Nat. Neurosci. 3(Suppl.), 1212-1217.

Wolpert, D. M., and Miall, R. C. (1996). Forward models for physiological motor control. Neural Netw. $9,1265-1279$.

Conflict of Interest Statement: The authors declare that the research was conducted in the absence of any commercial or financial relationships that could be construed as a potential conflict of interest.

Received: 22 November 2011; accepted: 20 February 2012; published online: 14 March 2012.

Citation: Kalckert A and Ehrsson $\mathrm{HH}$ (2012) Moving a rubber hand that feels like your own: a dissociation of ownership and agency. Front. Hum. Neurosci. 6:40. doi: 10.3389/fnhum.2012.00040

Copyright (c) 2012 Kalckert and Ehrsson. This is an open-access article distributed under the terms of the Creative Commons Attribution Non Commercial License, which permits noncommercial use, distribution, and reproduction in other forums, provided the original authors and source are credited. 